\title{
Distinct element analysis of inter-particle coating variability in a batch seed coater
}

\author{
Mehrdad Pasha $^{1, *}$, Mojtaba Ghadiri ${ }^{1}$, Alfeno Gunadi ${ }^{2}$, and Patrick M. Piccione ${ }^{3}$ \\ ${ }^{1}$ School of Chemical and Process Engineering, University of Leeds, United Kingdom \\ 2 Process Studies Group, Technology and Engineering, Jealott's Hill International Research Centre, Syngenta, United Kingdom \\ ${ }^{3}$ Process Studies Group, Technology and Engineering, Syngenta, Münchwilen, Switzerland
}

\begin{abstract}
Coating of particulate solids by a thin film layer is of interest in many industrial applications such as seed and tablet coating. In seed processing, seeds are commonly coated with a protective coating layer consisting of fertilisers and crop protection products. Rotary drum batch coaters are typically used for this purpose. The coater consists of a cylindrical vessel with a rotating spray disk in the centre, onto which the coating liquid is fed. The seeds are driven around the vessel by its rotating base, and are mixed by two baffles; one on either side of the vessel. In the present study, DEM simulations are used to analyse the seed coating process. Corn seed is used as a model material and its shape is captured using X-Ray microtomography (XRT). The shape is incorporated into the simulations by the clumping multiple spheres to form a particle assembly. The coating uniformity of the seeds is predicted by implementing a coating model, whereby the coating mechanism is represented in the DEM by considering that once a droplet contacts a corn seed, it is removed from the simulation and its mass is attributed to the coating mass of the corn seed. The distribution of mass of sprayed spheres on the corn seeds and the coefficient of variation are evaluated for a range of process conditions, such as spinning disk rotational speed, droplets size and baffle arrangement and designs. In addition to evaluation of coating uniformity of particles, the scale-up rules for rotary drum batch coaters are investigated and reported. The outcome provides guidelines on scale-up rules and improvement of coating uniformity for rotary batch seed coaters.
\end{abstract}

\section{Introduction}

Coating of particulate solids by a thin film layer is widely utilised in a variety of industrial applications including seed and tablet coating. In seed processing, rotary drum batch coaters are mainly used to coat the seeds with a protective layer consisting of fertilisers and crop protection materials. The effectiveness of the coating liquid formulation and the level of coverage of the coating on the seeds are important for optimisation of the seed coating process as these have a direct impact on the quality of the final product. Coating is influenced by motion, mixing of seeds, and liquid spreading over them, which are directly controlled by process operation.

The use of Distinct Element Method (DEM) [1] for the simulation of particulate systems has gained popularity in recent years since it provides a robust and realistic way of simulating the behavior of particulate matter during processing. More recently, it has been used to address the coating uniformity of pharmaceutical tablet coating in pan coaters [2-10]. There are two critical parameters for the analysis of the coating variability: inter- and intra- particle coating variability. The former is the variation in the average coating mass from a granule to another, whilst the latter is the distribution of coating liquid on the surfaces of individual granules, both of which can be quantified using the coefficient of variation [3].

The effect of seed coating process parameters (i.e. baffle angle and clearance gap to the wall, atomiser distance relative to the base and baffle geometry) on coating uniformity are investigated and their optimal values are identified. Moreover, the outcome of this study helps establishing scale-up rules for which little work has been reported in the literature.

\section{Materials and methods}

\subsection{DEM simulations}

A series of DEM simulations were performed for a rotary batch seed coater $(0.3 \mathrm{~m}$ in diameter and $0.21 \mathrm{~m}$ in height), as shown in Fig. 1, using EDEM $^{\circledR}$ software package (DEM-Solutions) to predict the impacts of various coating process conditions on the coating uniformity of corn seeds. Atomisation of a liquid stream introduced by a nozzle onto a spraying disc was simulated by generating spheres at a radial position (represented by a ring-shaped virtual geometry) corresponding to the tip of the rotating disc and at a rate conforming to the liquid flow rate. The droplet size and velocity distributions corresponded to the experimental

\footnotetext{
* Corresponding author: m.e.pasha@leeds.ac.uk
} 
values. In order to represent the coating mechanism in the DEM simulation, a droplet is removed from the simulation after it contacts a corn seed and its mass is attributed to the coating mass of the corn seed. The base rotates to mobilise the seeds, whilst the vertical plates act as baffles, turning the bed over and ensuring adequate mixing of the seeds in order to increase the uniformity of coating.

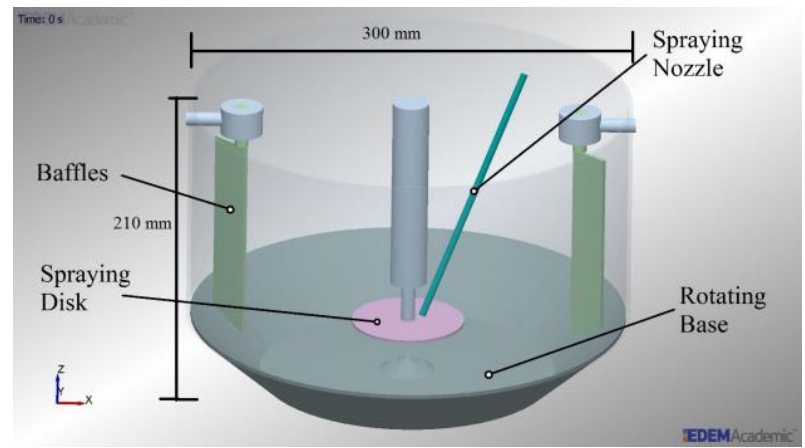

Fig. 1. Geometry of vertical batch seed coater.

\subsection{Particle shape}

A Phoenix Nanotom $^{\odot}$ CT scanner was used to obtain the three dimensional (3D) image of a single corn seed in the $7.1-8.0 \mathrm{~mm}$ sieve-cut, as shown in Figure 2a. The shape of corn seeds is then approximated with five clumped spheres (approximately $0.1 \%$ deviation in volume compared to volume of the actual seed) with different sizes, as shown in Figure $2 b$. The validity of the approximation for predicting the motion of corn seeds in the coater has been previously demonstrated by Pasha et al. [11]. A bed consisting of approximately 4200 seeds (21,000 spheres), corresponding to $1.4 \mathrm{~kg}$ of corn seeds, is used.
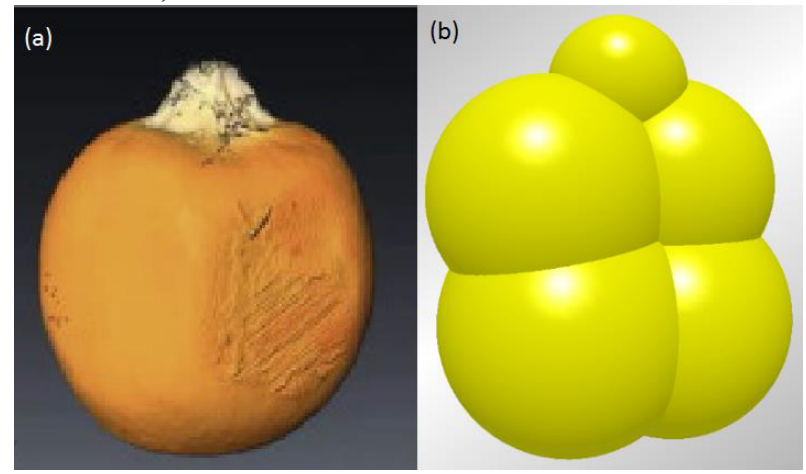

Fig. 2. (a) 3D captured shape of corn seed using XRT and (b) representation of corn seed shape by five overlapped spheres.

\subsection{Coating model}

The spray droplets are considered as non-interacting spheres until they contact the corn seeds. Video motion analysis is used to capture the motion of particles and determine their size and velocity distributions, which are then incorporated in the DEM simulations. In the simulation stage, a particle property termed 'coating mass' is introduced in addition to the existing properties. The model of Toschkoff et al. [10] is employed to model the coating process. According to this model, following a contact between droplets and corn seeds, the droplet mass is added to the coating mass of the corn seed and the droplet is removed from the simulation. The momentum of droplets is considered negligible compared to the corn seeds. As the impact of droplets on the corn seeds would not have a strong influence on the momentum of corn seeds, it is ignored in the force calculations. The Hertz-Mindlin contact model with rolling friction based on viscous dissipation [12] is used to simulate inter-particle interactions.

By taking into account the measured droplet size and velocity distributions and the direction of their movement, a custom code is implemented in EDEM software for generation and movement of droplets. A ring-shaped virtual geometry with $1.5 \mathrm{~mm}$ height and outer diameter corresponding to the disc atomiser diameter is considered as the particle factory for generation of the droplets. The droplets are randomly generated inside the particle factory and at the first time step of generation of each droplet; they are given an initial acceleration, which leads to the desired tangential velocity at the end of the subsequent time step. In the simulations, the rate of generation of droplet spheres is chosen such that their volumetric flow rate was equal to that used in the experiments, i.e. $2.67 \mathrm{ml} / \mathrm{s}$. This produces about 36,300 spheres per second. The coating simulations are done for 25 seconds of real time operation and the droplets are generated after one second of process time where the motion of seeds had reached steady state. The particle and simulation properties are summarised in Tables 1 and 2 where particle size follows a normal distribution.

Table 1. Particle and wall properties used in the DEM.

\begin{tabular}{lcc}
\hline Property & Seeds & Walls \\
\hline Particle diameter $(\mathrm{mm})$ & $7.5 \pm 3 \%$ & - \\
Shear modulus $(\mathrm{GPa})$ & 0.01 & 70 \\
Density $\left(\mathrm{kg} / \mathrm{m}^{3}\right)$ & 1163 & 7800 \\
Poisson's ratio & 0.25 & 0.3 \\
\hline
\end{tabular}

Table 2. Particle interaction properties used in the DEM.

\begin{tabular}{lcc}
\hline Property & Seed -Seed & Seed - Wall \\
\hline Sliding friction & 0.3 & 0.3 \\
Rolling friction & 0.01 & 0.01 \\
Coefficient of restitution & 0.6 & 0.69 \\
\hline
\end{tabular}

\section{Results and discussions}

\subsection{Baffle angle and wall clearance}

The effect of baffle angle and clearance to the walls on the coating uniformity of corn seeds are investigated by varying from $25^{\circ}$ to $55^{\circ}$ and $5 \mathrm{~mm}$ to $20 \mathrm{~mm}$, respectively. The latter is considered such that it represents a clearance corresponding to approximately 1 to 4 corn seed diameters. The definitions of the angle, $\alpha$, 
and clearance, $d$, are shown in Fig. 3. For all the investigated cases, the rotational speed of the coater base, atomiser disc distance to the base and flow rate of coating droplets are kept constant at $300 \mathrm{rpm}, 30 \mathrm{~mm}$ and $2.67 \mathrm{ml} / \mathrm{s}$, respectively. The coating simulations are carried out for $25 \mathrm{~s}$ of real time operation.

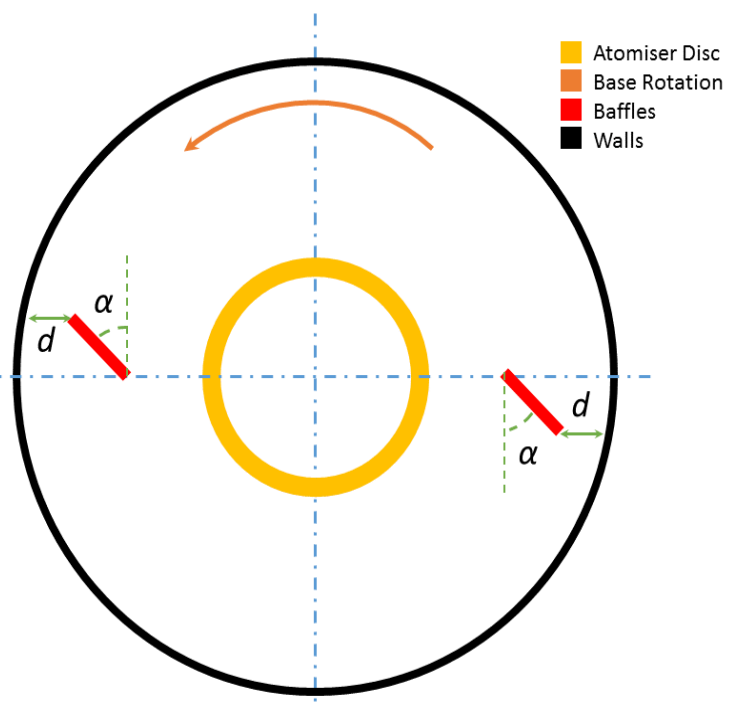

Fig. 3. Schematic representation of baffle angle and clearance gap to the wall.

The effect of baffle clearance to the wall on coating uniformity of corn seeds is shown in Fig. 4. For all investigated cases, the coating mass variability is reduced as the clearance gap is increased to $15 \mathrm{~mm}$, after which it increases again. Therefore, the optimum baffle clearance is around $15 \mathrm{~mm}$ for the investigated system here for all the baffle angles. This clearance represents approximately two to three particle diameters. The difference between the two extreme cases of clearance gap ( 5 and $20 \mathrm{~mm}$ ) investigated is considered significant, where $5 \%$ improvement in terms of coating uniformity is achieved. For clearance of 10 to $20 \mathrm{~mm}$, the coating uniformity is improved by increasing the baffle angle to $35-45^{\circ}$, beyond which the $\mathrm{CV}$ is slightly increased. However, in the case of a $5 \mathrm{~mm}$ clearance, the optimum baffle angle is found to be $25^{\circ}$.

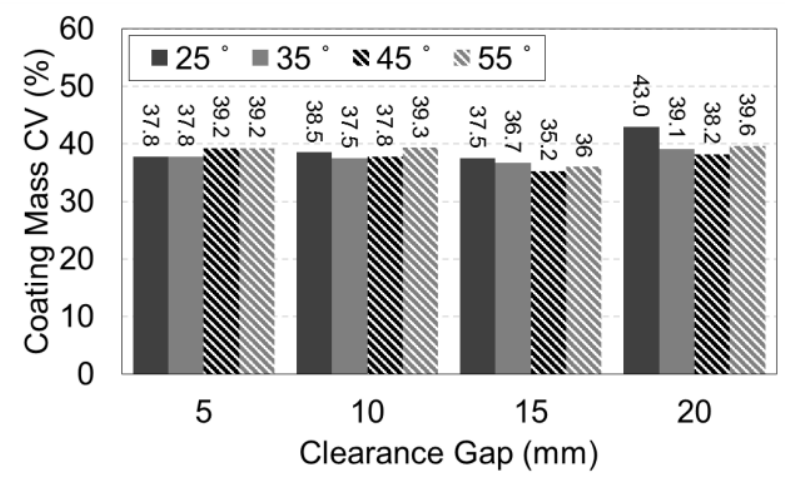

Fig. 4. Effect of baffle angle and clearance gap to the wall on coating uniformity of corn seeds in DEM simulations.
It can be concluded that optimum values of baffle angle and clearance to the wall of the coater are $45^{\circ}$ and $15 \mathrm{~mm}$, respectively, where the coating uniformity of corn seeds can be improved by $7 \%$ between the two extremes of coating mass $\mathrm{CV}$. The clearance gap to the wall controls the fraction of the surface that is renewed after each circulation of base, since this parameter controls the thickness of the particle layer being sliced off from the bed surface. Moreover, the baffle angle controls the direction and the position of the particles being sliced from the bed. In combination, these two parameters control the surface renewal rate of the bed, which directly influences the coating uniformity of corn seeds since only the particles at the surface receive coating liquid.

\subsection{Atomiser vertical position}

Since the coating droplets are generated using a rotating disc atomiser rather than a sprayer, a thin layer of droplets are generated. The position of the atomiser disc relative to the base of the coater affects the fraction of seeds being coated on the surface of bed. A series of DEM simulations were carried out by varying the vertical position of atomiser disc relative to the base of the coater from 30 to $70 \mathrm{~mm}$, using the flat design baffle (30 mm wide) with clearance and angle of $15 \mathrm{~mm}$ and $45^{\circ}$, respectively. The particle coating mass $\mathrm{CV}$ values using the proposed vertical positions of the atomiser disc are shown in Fig. 5. The coating uniformity of the particles improves as the vertical position is increased to $50 \mathrm{~mm}$ from the base; however, beyond this point it starts to deteriorate ( $\mathrm{CV}$ increases). Comparing the optimised vertical position of the spray disc $(50 \mathrm{~mm})$ with the initial position used in the earlier simulations $(30 \mathrm{~mm})$, the position of the disc relative to the base of coater has a notable influence on the coating variability and approximately $5 \%$ improvement of coating variability is achieved as shown in Fig. 5. Hence, it can be concluded that this process parameter is one of the key influential parameters in the coating process using this type of coater.

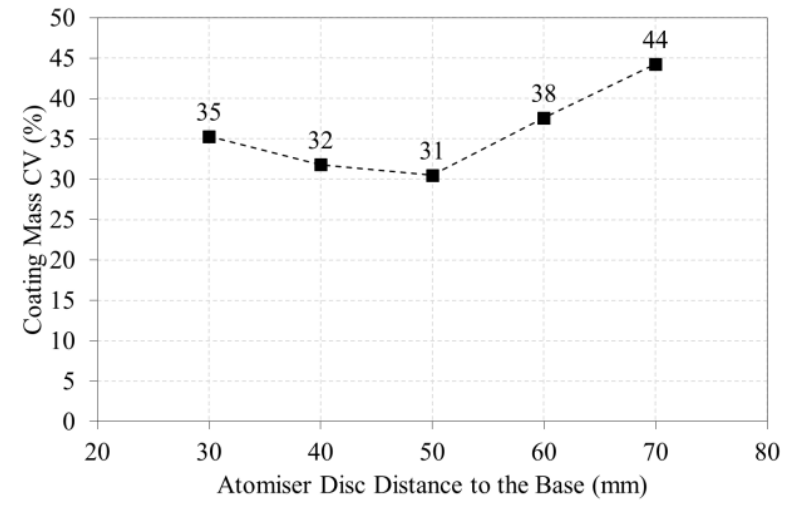

Fig. 5. Effect of atomiser disc position relative to the base of the coater on coating uniformity of corn seeds in DEM simulations.

\subsection{Rotational speed of the base}

There are a number of parameters that are likely to influence the coating variability of the particles in seed 
processing such as the dynamics of droplet generation, surface renewal rate, and number of coating events (e.g. number of bed turnovers). A larger number of rotations should lead to a higher probability of new and less coated particles being available for coating at the surface of the bed, hence leading to a higher probability that the particles will be more uniformly coated in the system. It is expected that a minimum number of bed turnovers is required to let all the particles become coated. However, an excessively high rotational speed would lead to higher impact velocities of the seeds to the baffles, which may damage both the particles and their coating.

In this study, DEM was applied to investigate the effects of various process parameters on coating variability of corn seeds. The focus here was on the coating variability of seeds rather than any damage on their coating. Since the potential damage to the coating of the seeds was to be avoided, only three base rotational speeds of 300, 400 and $500 \mathrm{rpm}$ were tried to investigate their effect on the coating variability. It was found that increasing the base rotational speed improved the coating uniformity of the corn seeds, but only very slightly, as approximately $1.4 \%$ improvement was achieved.

\section{Conclusions}

The effect of various process parameters on coating variability of corn seeds in a batch seed coater was investigated using DEM simulations. In the coating model, the coating liquid droplets were represented by small spheres. Once brought into contact with a corn seed surface, the mass of droplets was stored in the memory as the corn seed coating mass, and it was removed from the simulation. The uniformity of coating was then assessed based on coefficient of variation of the coating mass on each seed. It was shown that using this model the amount of the coating on each particle could be tracked; hence useful information such as distribution of coating mass among the particles and the coefficient of variation of coating mass of the particles could be assessed.

Among the investigated process parameters, it was concluded that the position of the atomiser disc relative to the base of coater and baffle clearance to the walls had a strong influence on the coating uniformity of the particles. In the case of baffle clearance to the wall, a clearance to the wall of $15 \mathrm{~mm}$ provided the lowest interparticle coating variability when compared to small and large clearance gaps. An improvement of approximately $7 \%$ was achieved between the best and worst case combinations of baffle angle and clearance gap investigated in this study. Additionally, it was found that the influence of other process parameters including baffles angle and base rotational speed on coating uniformity was insignificant since they only lead 1 to 2 $\%$ change in the coefficient of variation of the coating mass.

\section{Acknowledgements}

The financial support of Syngenta through its Technology \& Engineering's Strategic \& Enabling Technology programme is gratefully acknowledged. The authors would like to thank Dr Neil George, Syngenta Ltd, UK for his support and encouragement, and Phil Taylor of Syngenta Jealott's Hill Formulation Technology Group for providing the seed coater. The authors would also like to thank DEM Solutions for providing support and additional licences for the purpose of this work.

\section{References}

1. P.A. Cundall, O. Strack, Gotechnique 29 (2) (1979) 47-65.

2. H. Zhu, Z. Zhou, R. Yang, A.B. Yu, Chem. Eng. Sci. 63 (23) (2008) 5728-5770.

3. A. Kalbag, C.R. Wassgren, Chem. Eng. Sci. 64 (11) (2009) 2705-2717

4. A. Muliadi, P. Sojka, Atomization and Sprays 20 (7) (2010) 611-638

5. K. Yamane, T. Sato, T. Tanaka, Y. Tsuji, Pharmaceutical Research 12 (9) (1995) 1264-1268.

6. J. Park, C.R. Wassgren, Part. Sci. \& Tech. 21 (2) (2003) 157-175.

7. D. Suzzi, G. Toschkoff, S. Radl, D. Machold, S.D. Fraser, B.J.Glasser, J.G. Khinast, Chem. Eng. Sci. 69 (1) (2012) 107-121.

8. J. Li, C. Wassgren, J.D. Lister, Chem. Eng. Sci. 95 (2013) 203-210.

9. S. Just, G. Toschkoff, A. Funke, D. Djuric, G. Scharrer, J. Khinast, K. Knop, P. Kleinebudde, Int. Jour. of Phar. 457 (1) (2013) 1-8.

10. G. Toschkoff, S. Just, A. Funke, D. Djuric, K. Knop, P. Kleinbudde, G. Scharrer, J.G. Khinast, Chem. Eng. Sci. 101 (2013) 603-614.

11. M. Pasha, C. Hare, M. Ghadiri, A. Gunadi, P.M. Piccione, Powder Technology 296 (2016) 29-36.

12. Y. Zhou, B. Wright, R. Yang, B. Xu, A. Yu, Physica A 269 (24) (1999) 536-553. 